\title{
Non-Functional Properties in Service Oriented Architecture - A Consumer's Perspective
}

\author{
Hanane Becha and Daniel Amyot \\ EECS, University of Ottawa, 800 King Edward, Ottawa, ON, K1N 6N5, Canada \\ Email: \{hbecha,damyot\}@eecs.uottawa.ca
}

\begin{abstract}
Information about non-functional properties (NFPs) is rarely explicitly described in Service Oriented Architecture (SOA) services. In particular, there is still no standardized solution addressing what service providers should expose or advertise as NFPs in service descriptions to empower service consumers to decide whether a given service suits best their needs or not. Our goal is to define a catalogue of generic (i.e., domain independent) nonfunctional properties to be considered when service descriptions are developed. This catalogue should be used to better characterize services and enable consumers to perform advanced applications such as NFP-aware service selection. We have identified an initial catalogue of SOA-related NFPs that are relevant from the perspective of consumers (as opposed to providers). Then, we have designed an online survey and invited international SOA experts in many application areas to criticize the relevance and definitions of the proposed NFPs and to enhance this catalogue. After analyzing the survey results to synthesize an improved catalogue, we have validated the new definitions a second time with a subset of the initial participants. We obtained a validated list of 17 NFP definitions for atomic SOA service descriptions relevant from a service consumer's perspective.
\end{abstract}

Index Terms - Service Oriented Architecture (SOA), Service Description, Non-Functional Properties (NFPs), Consumer's Perspective.

\section{INTRODUCTION}

The Service Oriented Architecture (SOA) is an architectural style that promotes sharing and reusing software components in the form of published services [1]. While frameworks for managing non-functional requirements of conventional applications are well established [2], the corresponding frameworks in the context of SOA services are still in their infancy. In traditional software engineering, stakeholders play an important role in expressing explicitly or implicitly the non-functional requirements of their applications, and then in verifying and validating the implementation of these requirements at various stages of the applications' development life-cycle. However, in SOA, many service consumers are not involved in any stage of the development phase of the service. Services are used as black boxes (i.e., services are opaque), which means that service consumers do not have access to their underlying logic or implementation. Hence, service consumers cannot easily assess the services' behavioural and non-functional aspects until they actually invoke them. Thus, service providers should supply a detailed service description for each service. As well, in contrast with traditional application development processes, nonfunctional properties (NFPs) are not applied to and assessed against an application as a whole but against multiple independent distributed services, which brings up additional challenges.

In service-based applications, NFPs of services can drive the design of the applications (i.e., composite services) and the selection of the concrete services from which they are built. The accessibility to multiple services that can deliver the same functionality reinforces the need for formal NFP descriptions, since they may be the only differentiator between them. The ability of the service consumer to choose services based on the NFPs specified in the service description is crucial, along with the means to compare the NFPs of available services. In this paper, we focus our attention on the NFP description of atomic services, which do not rely on any other services.

At this time, non-functional properties are either simply not advertised or are described in proprietary ways. Service providers and service consumers need to have a mutual comprehension of, at least the basic, NFPs that should be exposed in the service description and the ways in which they are described. A catalogue of domainindependent NFPs that are important for services evaluation and selection is needed. Ideally, we should have a standardized catalogue of the NFPs of the SOA services. Our research hypothesis is that we can propose core generic NFPs, and their definitions, to be considered when service descriptions are developed, compared, and used. We believe that this catalogue should be extensible to include domain-specific NFPs for particular business areas. Specific business rules will drive the selection of appropriate NFPs to be considered out of the ones that we propose.

We have extracted an initial set of SOA-related NFPs from the literature and from work in progress in standardization bodies such as OASIS [3], W3C [4] and ITU$\mathrm{T}$ [5]. We have filtered the NFPs to keep only those that were relevant to the consumer's perspective, i.e., the NFP information that should be included in the service description, be measurable by the service participants, and help the service consumers decide whether the service suits their needs or not. For example, while NFPs such as cost and response time are key to consumers, other NFPs such as maintainability, profitability, and portability, 
while being very important to service providers, are not really relevant from a consumer's perspective. Then, we proposed a resulting catalogue of 19 NFPs (including their definitions and additional descriptions) as an online survey and invited participants to criticize and enhance these descriptions as well as to assess their relevance to the consumer's perspective in the SOA context. The participants were from industry, academia and standardization bodies. We have analyzed the survey results to compile an improved catalogue. We have reiterated the process by resubmitting the resulting catalogue to the survey participants that had expressed their will to further collaborate. Subsequently, we have analyzed the new comments and concluded with a final set of 17 NFPs and definitions that we present in the following sections of this paper.

These contributions are both important and timely for industry. For instance, the Object Management Group (OMG) [6], whose members are major players in the software, telecommunication, and service areas, have issued a Request for Information (RFI), which is the first stage of OMG's standards development process, on the proper handling of NFPs in SOA [7].

This paper is structured as follows. Section II reviews relevant background concepts and related works on services, quality, ontologies, NFP catalogues, and servicelevel agreements. Section III presents the survey methodology, whose results are discussed in Section IV. Our revised list of 17 consumer-oriented NFPs for atomic SOA services is summarized in Section V. Additional observations can be found in Section VI. Finally, Section VII presents conclusions and items for future work. Note also that Appendix A provides a useful list of acronyms and abbreviations used in this paper.

\section{BACKGROUND AND RELATED WORK}

\section{A. Services in $S O A$}

SOA is an architectural style that promotes sharing and reusing software components (i.e., published services). Services are discoverable as service providers publish their services' descriptions in registries. Service consumers can then discover, select, and invoke or compose these published services to meet their business needs.

SOA is not tied to any specific technology and does not rely on any particular implementation, although it is commonly implemented using web services, being primarily developed by the Organization for the Advancement of Structured Information Standards (OASIS) and the World Wide Web Consortium (W3C).

As defined in the OASIS reference model for Service Oriented Architecture, "SOA is a paradigm for organizing and utilizing distributed capabilities that may be under the control of different ownership domains. It provides a uniform means to offer, discover, interact with and use capabilities to produce desired effects consistent with measurable preconditions and expectations" [1]. The part emphasizing "under the control of different ownership domains" is of a particular interest. It implies the ability to use services provided by third parties, which often speeds up applications' development time in com- parison with present long-established, tightly-coupled, embedded environments. However, when service providers and service consumers are not within the same organization, service descriptions could be the only means to "communicate". Service consumers do not have to develop or even understand the underlying logic and implementation details of services they use. Services abstract form their underlying logic, which means they share nothing but a formal contract that contains only the information required by service consumers to determine whether a given service is appropriate for their needs (including functional and non-functional properties of the service) and the information necessary to interact with the service such as service interfaces, behavior, and location.

\section{B. NFPs and Qualities in SOA}

Nowadays, there is still no complete standardized solution specifying what service providers should expose as NFP information in their service descriptions. We stress the fact that the focus is not on all the NFPs of the SOA service but only the subset of the NFPs of interest to the service consumers: the consumer's perspective.

In the literature, non-functional requirements are often referred to as qualities of an application. Other terms often used for non-functional requirements include constraints, quality attributes, quality goals, and quality of service requirements.

In our survey, we presented an initial catalogue composed of 19 NFPs (cost, completion time, trust, availability, reliability, usability, validity, standards compliance, dependencies, failure mode, security, execution models, jurisdiction, life-cycle updates, penalty rate, compensation rate, resource utilization, throughput, and accessibility) to describe common non-functional characteristics. First, an initial set of SOA-related NFPs was extracted from literature and from work in progress in standardization bodies such as OASIS, ITU-T and OMG. Second, we have filtered the NFPs to keep only those that were relevant to the consumer's perspective.

Part of the literature work that we have considered when extracting our initial set of NFPs includes the following:

- The Quality Model part of the ISO/IEC 9126 [2], which is an international standard for evaluating the quality of conventionally (i.e., non-SOA) developed applications. Its taxonomy, defined for traditional software engineering, is not entirely relevant for SOA, where the NFPs are often defined without involving the service consumers.

- The Quality Model Working Draft (WS-Quality factors) [8][9], initiated in September 2005 by OASIS with the aim to define NFPs specifically for web services technology. This document provides guidelines and detailed implementation notations for the service provider. It aims to define and formalize a set of attributes in the context of contracting for web services. It is still incomplete (i.e., not officially published yet) and rather tied to web service technologies. In addition, it is not focused on service description from the perspective of the service consumers. 
- Choi et al. [10] have identified some of the unique features of SOA and then derived six quality attributes and their respective metrics to measure each attribute. The proposed set of attributes is intended to be used by service providers to ensure that a qualified service is published. This is a focus different than ours.

- Glaster et al. [11] recognize the critical importance and the difficulties associated with handling NFPs in general and the fact that they are even more difficult to handle in the SOA context. They attempt to generate a checklist of NFPs for SOA to be used by the service providers. Their NFPs are organized into three categories: process requirements, non-functional external requirements, and non-functional service requirements. The authors provide an extensive generic list of various NFPs to be considered when services are under development. However, they provide only informal information on the measurability of these NFPs.

- Balfagih and Hassan [12] examine the quality of service (QoS) of SOA and web services, and classify them from the perspectives of the developer, provider, and consumer. Their list of QoS from the perspective of the consumer includes response time, availability, reliability, security, usability, composability, and robustness. They define the three first QoS and propose corresponding metrics. For security, they just mention a few sub-factors including confidentiality, integrity, authentication, and availability. They define usability but again they do not propose any metrics; they simply mention sub-factors including understandability and configurability. Composability was initially part of our catalogue but, following various discussions, it was removed since the essence of SOA is the capability of composing services in order to create more complex ones. Balfagih and Hassan define composability but they do not provide metrics. They propose the following sub-factors that facilitate composability: conformability, availability, discoverability, and the loosely coupled nature of the web services. These sub-factors are the ones proposed in their model for the developer's and provider's perspectives. Robustness, also referred to as flexibility, is not part of our catalogue; their definition relates to our usability NFP (the service works even when the input is somehow not valid). Balfagih and Hassan's paper provides a good literature review and QoS classification. However, there is no validation methodology or justification of the selected or eliminated QoS. Formal definitions and metrics are provided only for a few QoS.

\section{NFP Ontologies and XML-Based Languages for SOA}

The popular Web Services Description Language (WSDL) is meant to describe the functional aspects of a service. When this language was developed, it did not aim to describe the NFPs of the service. To support management of web service and their dynamic composition, several languages are being developed (e.g., Web Service Offerings Language [13] and Semantic Markup for Web Services [14]). In a nutshell, using XML, these languages formalize various constraints (e.g., policies) for web services, including those related to NFPs. To complement these efforts, NFP ontologies are needed, and several have been proposed:

- The Semantic Markup for Web Services (OWL-S) (formerly the DARPA Agent Markup Language, DAML-S) [14] is an XML-based language for semantic descriptions of web services. It provides a declarative language for service properties and capabilities advertisement, including placeholders for NFP descriptions. However, OWL-S does neither propose an exhaustive list of NFPs to be exposed as part of the service description nor NFP metrics to enable the comparison of NFPs.

- Web Services Policy (WS-Policy) [15] is a general framework specifying policies for web services. The details of the specification of particular categories of policies (including NFP definitions) need to be defined in specialized languages.

- UDDI eXtension (UX) [16] empowers the Universal Description, Discovery and Integration (UDDI) registries to collect feedback from the service consumers on their satisfaction about the NFPs of the invoked services, and to compute the average satisfaction. This average satisfaction is used predict the performance of the service in the future. Their NFP list is limited to response time, cost and reliability.

- In Web Service Modeling Ontology (WSMO) [17], the authors sketch some domain-specific NFPs from the functional point of view of the web services. The recommended set of NFPs include the following: accuracy, contributor, coverage, creator, date, description, financial, format, identifier, language, network-related QoS, owner, performance, publisher, relation, reliability, rights, robustness, scalability, security, source, subject, title, transactional, trust, type, and version. These NFPs are just mentioned, without really being defined.

- Zeng et al. [18] have defined a QoS-aware middleware for web services composition. First, the NFPs are defined for atomic services including execution price, completion time, reputation, successful execution rate and availability. The catalogue of NFPs is actually very restricted. Second, a NFP aggregation model for a composite service is expressed. We focus on the former part in our paper.

- Dobson et al. [19] recommend the use of domainindependent ontologies to represent, organize and reason about NFPs in SOA, hence unifying the concepts and terms used throughout the service lifecycle. They propose a NonFunctionalProperty ontology that consists of two hierarchies - one rooted at the class QoSAttribute and one rooted at the class QoSMetric. To support measurable non-functional requirements, these two hierarchies are joined together by an OWL ObjectProperty named hasMetric. They also differentiate QoSAttribute from other non-functional properties that are not considered as QoS (e.g., cost). To increase the flexibility of comparing the NFPs of different services, this ontology defines explicit semantics 
that allow the automatic conversion between compatible units and metrics. For example, service availability can be stated as the Mean Time Between Failures (MTBF) and Mean Time to Recover (MTTR). If we define a rule in the ontology statins that availability = MTBF / (MTTR+MTBF), then the discovery component can still correctly compare queries stated in terms of availability. Dobson et al. have demonstrated that the use of an ontology in the specification and discovery of the services provides a greater degree of machine understanding than the current NFP languages.

- Tosic et al. [20] define a Web Service Offerings Language (WSOL) to formally describe different classes (or profiles) of service (e.g., services refer to the same WSDL but have different levels of QoS constraints and management statements). A service is defined as various formal constraints (e.g., functional constraints, QoS, and access rights) and management statements (e.g., prices, monetary penalties, and management responsibilities). WSOL along WSDL could be used to support Web Service Management and Web Service Composition Management applications. Tosic et al. confirm the importance of NFPs in service selection and composition, and they developed mechanisms to monitor and adapt NFPs to enable dynamic service management. However, WSOL does not define QoS constraints to be monitored but uses external ontologies of NFPs. Tosic et al. share our opinion on the need for ontologies that contain precise definitions of the NFPs metrics and how they should be measured. They agree as well that the ontological definitions should be domain independent. Also, we are both in agreement that ideally appropriate standardization bodies would develop such ontologies to facilitate interoperability. However, due to the standardization process, which is often very time consuming, it could be practical that this work be initiated by other interested parties. Our NFPs catalogue is in line with this work and it does complement it as a needed piece of the WSOL puzzle.

The WSOL paper [13] highlights the need for ontologies that define and represent formally QoS/NFP metrics. These ontologies are needed to specify QoS constraints for languages used in the management of web service and their dynamic compositions. Tosic et al. depict the requirements of such ontologies, which include having QoS metrics, measurement units, currency units, measurement methods, measured properties, and dependencies and relationships between QoS metrics. Based on their study of existing ontologies, including the DAML Ontology Library [21], the Cyc Upper Ontology [22], and the Simple HTML Ontology extensions (SHOE) [23], they confirm the non-existence of any satisfying NFP catalogue. Our work contributes to filling the gap in these existing ontologies.

\section{NFP Catalogue of O'Sullivan et al.}

Publications from O'Sullivan, Edmond, and Hofstede [24][25] are in line with our vision on the need for a basic set of domain-independent non-functional proper- ties that can be used to improve discovery, comparison and service substitution. In [24], Edmond et al. highlight two concerns with the existing approaches to the NFP description of web services. The first concern is a "Web service tunnel vision" and endorses the need to utilize lessons learned from conventional services description. We have no problem with this statement. However, we think that we should have a broader vision since SOA brings new challenges that we did not have in the context of conventional services. In addition, one can argue the need to compare conventional service versus a web service. Nowadays, service requestors do not have Internet phobia anymore and SOA-based services are becoming very convenient (e.g., in terms of price, speed and availability) when compared to conventional services. Their second concern raised is called the "Semantic myopia" and is defined as not taking advantage of the semantic richness of NFPs. We agree that NFP descriptions in SOA should take advantage of all the existing work related to non-functional requirements and should be leveraged to address the new SOA challenges.

The large technical report by O'Sullivan et al. [25] contains 79 models that describe NFPs covering the following: availability, payment, price, discounts, obligations, rights, penalties, trust, security, and quality. The authors have done a comprehensive work on the importance of NFP detailed descriptions as a motor to improve discovery, comparison and service substitution. Their methodology is based on the review of existing commercial services, including mainly conventional ones. They outlined the importance and the complexity related to handling of NFPs and highlighted the issues associated with the rich NFP descriptions. In fact, the description of NFPs is indispensable for service life cycle (e.g., service discovery, substitution, composition, and management).

We obviously cannot review these 79 proposed models in this paper, but they were all considered for the initial list used in the survey and they will be revisited again for the design of metrics measuring NFPs. Note that some of their NFPs have the same names as ours but with totally different definitions. In [25], they define availability as temporal (i.e., when) and spatial (i.e., where for locationbased services). We believe that Internet-based services are being available 24/7 and in seamless way. For example, no matter where you are or the time of the day, you can still access your bank account online. Whenever, the region or the country is important, we have another NFP in our catalogue (i.e., jurisdiction) that addresses this.

In their catalogue, the NFP quality is defined as compassing 5 dimensions as follow: reliability (e.g., dependability and accuracy); responsiveness (e.g., promptness and willingness of staff to assist); assurance (e.g., courtesy of the staff); empathy (attention provided to the requestor); and tangibles (e.g., concrete aspect of the service such as cleanness). In comparison to our catalogue, quality is defined in a very subjective way and sometimes with criteria not even relevant to SOA services (e.g., assurance and empathy).

In addition, O'Sullivan et al. are neither focused on the service consumer's perspective nor clear about the sepa- 
ration between contracts and SLAs versus service description. We note as well that they do not differentiate mutually negotiated and agreed-on SLAs (e.g., referred to as settlement models) versus multiple possible settlement models proposed by providers allowing consumers to pick the one that matches best his or her needs.

\section{E. NFPs and Service Level Agreements}

At this point, we need to clarify common confusion when discussing NFP descriptions. Many will suggest using SLA templates as NFP descriptions. However, we stress that an SLA is a custom-made, negotiated (and potentially re-negotiated), long-term contract between two specific participants (service provider and service consumer). The information contained in a service description can be seen as a simple version of an SLA and/or an indispensable part of an SLA. Using only service descriptions, the provider can propose several services and the consumer picks the one that seems the most appropriate for his/her needs, when he/she needs it.

Some of the NFPs proposed in the previous sections, in our opinion, are closer to what is found in SLAs than to a consumer-oriented service description. Our experience (and that of our survey participants) showed that penalties for example should not be part of the service description since this aspect may change more often than the service NFPs, and penalties may be different for different services consumers. In some context, it is almost impossible to predict all scenarios and delineate the responsibilities of each participant in the service chain without human intervention. Using precise service descriptions requires less operating expenses, effort and time then human-negotiated SLAs. On the other hand, SLAs can be more practical in situations where providers have numerous service consumers (i.e., mobile phone subscribers) or for mission-critical applications.

For both consumer-oriented service descriptions and SLAs, having a detailed NFP ontology enables service comparison and selection. The majority of current works define SLAs for XML-based web service. These languages make many assumptions about the infrastructures at the service provider and service consumer sides. As a result, these specifications are neither easy to be reused in other contexts and infrastructures, nor easy to be extended for specific domains. Among these languages, we find the Web Service Level Agreement (WSLA) [26] defined by IBM and the Web Service Management Language (WSML) [27] defined by HP. They define their built-in NFP metrics, which make these NFP lists hard to extend in some cases while causing substantial overhead in some scenarios. Another language that can be used for specification of SLAs for web services is SLAng [28]. Again, the definitions of NFP metrics are built into the SLAng schema. Consequently, SLAs have a predefined format that hurts flexibility.

The Web Services Agreement Specification (WSAgreement) [29] from the Open Grid Forum (OGF) is a formal specification of agreements and agreement templates using XML, for grid services. Grid services can be seen as a special case of web services. The agreement (or SLA) still needs to reference the service description, including the NFPs, which will be delivered under the agreement.

Notably, to the best of our knowledge, none of the papers was dedicated to define a domain-independent catalogue of NFPs to accommodate the perspective of the service consumer. Our contribution is to filter and validate the superset of proposed NFPs to tackle the concerns of service consumers in the SOA context and independently from SOA-enabling technologies.

\section{SURVEY Methodology}

\section{A. Survey on NFPs for SOA Services}

In 2010, we have conducted a survey entitled "NFPs of services in SOA - Service Consumer's Perspective". The purpose of this survey was to discover requirements for an extensible framework to better describe NFPs of services in SOA purely from the point of view of consumers. We have proposed a catalogue of a 19 NFPs that can be included in service descriptions in order to enable advanced applications such as NFP-aware service selection. We invited people to share their opinion about the NFPs mentioned above to be exposed as part of the description of a service. We aimed to identify the generic NFP information that should be included in the service description to empower the service consumers to decide whether a given service suits their needs or not.

Only basic knowledge of SOA principles was required to the level of understanding the possible needs of service consumers. Therefore, no experience with any specific SOA implementation technology was necessary. The input of the participants was used to evaluate and evolve our proposed catalogue of service NFPs. The outcome of the questionnaire was collected and used anonymously in our research.

The survey consisted of four major parts. Part one explained the context and the goals of the survey and provided consent information to the participants. The second part was about participant characterization via six demographic questions. The third part proposed 19 NFPs with their meanings and provided text boxes inviting the participants to review, comment, criticize and improve the NFP descriptions. The fourth part was dedicated to the prioritization of the proposed NFPs into 4 levels (Absolutely essential, Might be needed, Not sure, or Definitely not needed) and to the collection of suggestions about other NFPs to be considered. The complete questionnaire is available online, together with the list of comments and suggestions provided by the participants [30].

The invitation to participate was sent mainly to the mailing lists of important standards development organizations including the Object Management Group (OMG), the Organization for the Advancement of Structured Information Standards (OASIS), and the International Telecommunication Union - Telecommunication Standardization Sector (ITU-T), as well as to a number of professors of the University of Ottawa and Carleton University (Canada) known to work on SOA. The survey and the answers were all developed in English.

Twenty-nine (29) respondents have completely answered the survey (53 people started it but many did not 
answer the questions beyond the demographic ones, so only those who answered all questions were counted).

\section{B. Initial NFP Catalogue}

The initial catalogue initially proposed in the survey is composed of following 19 NFPs: cost, completion time, trust, availability, reliability, usability, validity, standards compliance, dependencies, failure mode, security, execution models, jurisdiction, life-cycle updates, penalty rate, compensation rate, resource utilization, throughput, and accessibility. Their complete initial definitions are available in the original questionnaire [30]. As most of these NFPs are redefined after validation in the next section, we include here only the definitions of the NFPs that will not be redefined in the revised catalogue:

- Dependencies contain the list of other required services on which the execution of a given service depends, if any. This applies to a composite service that is built from other services.

- Execution model: the service should expose whether it supports a publish/subscribe model (i.e., eventdriven SOA) or uses a request/reply model (i.e., conventional SOA).

- Penalty rate is the fee that a service consumer has to pay to a service provider whenever he breaches the contract.

- Compensation rate is the fee that a service provider has to pay to a service consumer whenever the service provider was not up to the promise defined in the service agreement.

- Accessibility is the possibility of providing access to information to people with disabilities and people with special needs.

\section{Second Round of Validation}

After having collected the comments of the participants and having addressed them, a revised version of the NFP catalogue was produced and sent by email to a subset of the participants (i.e., eight people who provided an email address to indicate their interest in further collaborating to this validation exercise). Based on this second round of feedback, additional changes were incorporated to produce the final list of 17 consumer-oriented NFPs for atomic SOA services reported in Section V.

\section{Threats to Validity}

We are aware of three important threats to the validity of our results. First, the participants are mostly from the service provider's side (i.e., solutions developers, mainly into B2B and SLAs), hence, they are not necessarily the best people to represent the service consumers perspective. Second, the survey tool does not use a feature to randomize the ordering of the questions (so some bias might be introduced here). Third, the questionnaire is lengthy and requires a fairly high concentration; participants may get tired or simply rush through the last few NFPs/questions. The 24 participants who started the questionnaire and gave up quickly after a few NFPrelated questions suggests that the survey was indeed not easy to answer (we also suspect that several people were simply curious about the content of the survey). We tried to mitigate this last threat by providing feedback on the progress of the participant as he/she answered the survey questions.

\section{RESULTS}

\section{A. Demographic Information}

The survey was widely promoted, and 29 complete sets of responses were provided from around the world. The answers to the first six questions present a summary of the participants' demographic profiles.

Question 1: Are you from academia, government, or industry? 20 participants were from industry, 5 from academia, and 4 from the government.

Question 2: How many years of software development/teaching experience do you have?

- 13 participants answered more than 20 years;

- 9 had between 11 and 20 years;

- 3 had between 6 and 10 years;

- 2 had between 2 and 5 years; and

- only 2 had less than 2 years of experience.

Question 3: What is the level of your expertise in SOA? 10 participants stated they were experts, 14 said intermediate, and 5 were beginners.

Question 4: What is the primary focus of your organization? The participants were from: Consulting (7), Telecommunications (6), Education (5), Security (2), Software Engineering (2), Government (2), Standardization (1), and Other (4).

Question 5: What stage is your organization currently in with respect to SOA? The participants indicated: Implementing (10), Using (9), Investigating (6), Planning (1), None of the above (3).

Question 6: How long has your organization been caring about SOA?

- 7 participants answered more than 5 years;

- 16 said between 2 and 5 years;

- 2 stated less than 2 years; and

- 4 said that they were not interested yet.

Keeping in mind that participation to this survey was totally voluntary and unpaid (e.g., done for free), these answers show that we have got a reasonable and quite diverse participation, considering multiple dimensions, with a majority of participants from the industry (e.g., 20 participants out of 29) working in at least 8 different sectors. In addition, more than $2 / 3$ of the participants have more that 10 years of experience and almost half of the participants have more than 20 years of experience. A third of the participants consider themselves experts in SOA. The majority of our participants are implementing or using SOA, facing and dealing with SOA real-life problems. Only 4 of our participants are involved with organizations that are not yet interested about SOA. We therefore believe that we have got an appropriate, decent, and skillful participation that provides confidence to our validation results. 


\section{B. Key Results and Findings}

The participants were asked to review and prioritize the set of provided NFPs (the relevancy of the NFP, the label, and the corresponding definition). In addition, they were asked to propose new NFPs when this was deemed essential.

We have systematically taken all of the comments received into account, and decided together whether to incorporate or reject them. All the individual comments and resolutions are documented online [30].

With this first round of inputs, we ended up renaming many of the proposed NFPs, removing others, and adding new ones. All the proposed definitions were reworked and refined to address the main concerns raised by the participants and summarized as follow:

Separation of responsibilities: service providers will not commit to things out of their control. The participants were clear about the need to separate the responsibilities of the service providers, service consumers, and network providers. Hence, it is deemed necessary to have NFPs that depict the service requirements from the consumers' side (e.g., resources requirements). As well, we had to separate the network latency from the providers' promised response time and accordingly the network availability and reliability from the service availability and reliability.

Concise definitions: The participants have expressed the need to keep the definitions concise and separate from any supportive text. As a result, we have added a note for our supportive text, including information about possible metrics and semantics for the NFPs.

NFP names: Participants were very meticulous about the NFP names. Hence, we renamed multiple NFPs to address the concerns raised about any possible ambiguity. In the final list of NFPs, cost becomes price, completion time becomes response time, trust becomes reputation, validity becomes accuracy, life-cycle updates becomes service versioning, resource utilization becomes resource requirements, and throughput becomes scalability.

New NFPs: We did stress the difference between what the service providers claim to offer and what could be certified by other organizations. Hence, we have added a new NFP: certification. We have also added two other NFPs: transactional service, and server location. Transactional service is justified when services involve a sequence of independent updates that must be committed as a logical unit. Services may need to participate in transactional two-phase commits and a transactional management may need to be spread across invoked services for composite services. Server location is required for some participants who do not want their data to enter a given country.

NFP prioritization and deleted NFPs: According to the prioritization results (expressed in terms of percentage of participants for the categories Absolutely essential / Might be needed / Not sure / Definitely not needed), four NPFs received overwhelming support: availability $(80 / 20 / 0 / 0)$, security $(83 / 13 / 3 / 0)$, completion time $(73 / 23 / 3 / 0)$, and reliability (83/10/7/0). Although none of the NFPs received more than two "Definitely not needed" verdicts, five NFPs received much hesitant support, both in terms of prioritization ranking and of textual comments. Accordingly, we ended up removing these NFPs:

- Penalty rate (23/27/43/7), as it should be part of an SLA. Such rate should be negotiated and agreed on after having a clear identification of the causes of the problem at the service level.

- Compensation rate (23/30/40/7), removed for the same reasons identified for penalty rate.

- Dependencies (23/43/27/7), as this NFP does not apply to atomic services. It may be considered in the future for composite services.

- Execution model (23/43/27/7), considered as a functional property.

- Accessibility (23/43/27/7), argued as being a user interface property rather than an SOA property.

Domain independence: We aimed to define a domainindependent catalogue of NFPs. Having participants from different fields helped us tailor the definitions to make them general enough to cover many domains while remaining small enough to avoid too many domain-specific particularities.

\section{CONSUMER-ORIENTED NFPS FOR SOA}

The final list of consumer-oriented NFPs for SOA atomic services is composed of the following seventeen properties: (1) price, (2) response time, (3) reputation, (4) certification, (5) availability, (6) reliability, (7) usability, (8) accuracy, (9) standards compliance, (10) failure modes, (11) transactional service, (12) security, (13) jurisdiction, (14) service versioning, (15) resource requirements, (16) scalability, and (17) server location. Note that the order in which these NFPs are presented in this section is arbitrary. In addition to a concise definition, explanation notes as well as relevance and measurability information are provided for each NFP.

1. Price is the fee that the service consumer is expected to pay for invoking a given service.

Note: Different pricing models can be supported including: fixed price per invocation, calculated by a function based on the input service request (e.g., when a "back-up storage" service is requested, the price could be calculated based on the total size of the files and on the storage duration), per subscription (e.g., "all you can use" for a given period), or a mixed model (e.g., having service invocation fee on top of a subscription fee). Service providers can offer functionally equivalent services for different pricing models. These services are functionally equivalent services which mean the same class of service but different services that we treat separately.

Relevance: This is an important criterion since organizations and consumers operate accordingly to their financial plans.

Measurable: Two parameters to expose the cost of using a given service: first, how much to pay (and in which currency), and second, which mode of payment (per invocation or for a certain quantity such as a given duration or size). 
2. Response Time is the duration of the execution of a given service.

Note: Response time can be expressed as the probability of executing the service during a certain number of time units. Many duration-probability pairs can be used. Service request processing under a certain workload is considered, but not network delays (the latter being out of the control of the service provider). For example, process queries in less than 30 seconds $95 \%$ of the time. Response time could be affected as well by the input of the invoked service (e.g., the size of files that the "backup storage" service is invoked against). When the response time is bigger than the acceptable bound, then the service is considered as being not available.

Relevance: Time is a common measure of performance. Organizations are enhancing continuously their processes to be more responsive to the needs of their customers. Shorter service execution time is a competitive advantage allowing delivering rapidly more value.

Measurable: This can be expressed in terms of time units that are observable by the service consumer minus a probabilistic time units for the network delay (i.e., network delay is not included in the response time).

3. Reputation is the opinion of service consumers toward a service.

Note: Reputation can be expressed as the average ranking (feedback) of the service consumers of the service. Level 0 to level 5 (i.e., [0..5]) can be used, the latter being the best score (e.g., the highest satisfaction). Reputation is populated by previous service consumers and exposed by the service providers. Security mechanisms can be putted in place by the service registry to ensure that only consumers can enter that ranking of the service reputation.

Relevance: Reputation is a subjective perception of service consumers toward a service. It tells us nothing about how the service was tested or how secure it is. Still, reputation is a key criterion in establishing a functioning relationship between service providers and service consumers; hence the transactions can take place. A service consumer will not do business unless he understands what he is getting for his money, the terms and conditions of the service provisioning, and the reputation of the provider. The service provider needs to be assured of the consumer's ability and willingness to pay for the service in a timely fashion.

Measurable: This is based on the perception of the service consumers.

4. Certification is the confirmation of certain characteristics (i.e., affiliation, signature, specification, policy, standard, law, regulation, etc.) of a tested service by a certification body. Generally certification is provided by some form of external review, audit, or assessment performed by an independent entity.

Note: Certification gives greater guarantees of compliance. Certification can be expressed as many Characteristic-Level pairs. For example, Common Criteria certifica- tion (ISO/IEC 15408) specifies to what Evaluation Assurance Level (EAL1 through EAL7) the service has been tested. Certification is ensured and populated by third-party organizations and exposed by the service providers.

Relevance: It is deemed necessary to have independent third party tester of certain aspects of the service. For example, under the Common Criteria, a vendor must submit their products for a third party to test and grant some level of assurance from level one to level seven (very high level of assurance).

Measurable: By definition, certification is ensured by a given certification body.

\section{Availability is readiness for correct service.}

Note: Availability can be expressed as the probability that a service is accessible and operational when required for use at a given moment. Even if the service is available but its response time is bigger than the acceptable bound, the service is considered as not being available. Availability computation should be based on unscheduled downtime. In an environment with a dynamic infrastructure, there can be variable probabilities of availability. Availability may vary, in a known way, as a function of the time of day, day of the week, day of the month, etc. Hence, probabilities may need to have associated time boundaries. Many percentage-duration pairs can be used. The availability of the network is out of scope.

Relevance: Availability is a well-established criterion in the literature. Downtime per year is a very intuitive way of understanding the availability. Poor availability can cause a loss of business opportunities and slow down productivity. Service providers that publish their predicted availability numbers will help them gain an advantage over their competitors who either do not publish their numbers or have lower numbers.

Measurable: Availability is computed as 1 - (Down Time / Measured Time).

\section{Reliability is the continuity of correct service.}

Note: Reliability can be expressed as the probability that a service, after being invoked, operates correctly (i.e., without failure or unacceptable degradation of performance) under given conditions for a given interval of time. Many percentage-duration pairs can be used. The reliability of the network is out of the scope.

Relevance: Reliability is a well-established criterion in the literature. A service provider's reputation is very closely related to the reliability of their services. Reliability is very important for service consumers, since it is a mandatory requirement for their satisfaction. For service providers, if a service fails to perform its function, this will negatively affect their profits. As well, a high reliability is an important competitive advantage for service providers since their services will require fewer debugging and/or less maintenance. Service providers that publish their predicted reliability numbers gain an advantage over their competitors who either do not publish their numbers or have lower numbers. 
Measurable: Reliability is computed as Number of Successful Executions / Total Number of Invocations for a given interval of time.

7. Usability is the capability of the service (when used under specified conditions) to be understood by, learned by, used by, and attractive to the user.

Note: Usability can be calculated as the average ranking of the feedback provided by the service consumers regarding time to become proficient, frequency of resorting to help, and frequency of rework. Usability Level 0 to Usability level 5 (i.e., [0..5]) can be used, the latter being the best score (e.g., best experience of use). Note that usability may be inapplicable to some services, for instance when a service does not interact directly with a human being. Hence, the usability property must also state whether it applies to the service or not. Usability is populated by previous service consumers and exposed by the service providers.

Relevance: Usability began to obtain the recognition back in the 1980s when the importance of usability was acknowledged. If a service is not easy to use, then the service consumer will not feel confident to invoke it, in particular when other functionally-equivalent services are available and easy to access. No matter how good the service will perform its functionality, the service consumer has to be comfortable to invoke it first. High usability results in increased revenues for service providers and service consumers. For service consumers, usability will improve their productivity, and reduced their errors. For a service provider, usability can return significant benefits on investment with increased service consumers' attraction and retention.

Measurable: This is based on the perception of the service consumers. A mechanism to ensure that only service consumers can enter this information should be in place.

8. Accuracy is the degree of proximity of a measured or calculated result to its true result.

Note: Accuracy applies only to services that provide information (or data). Accuracy can be expressed as a percentage.

Relevance: The level of accuracy required for particular applications varies greatly. It is recognized that inaccuracy can "make or break" many types of services. While inaccuracy can make the results of some services almost worthless, excessive accuracy is not only costly but can cause considerable overhead and delays. For example, for a GPS location service, the order of magnitude will be meters and the time will be hours. Accuracy could be specified in terms of percentage.

Measurable: Invoking the service using an input for which the output is known and comparing the results.

9. Standards Compliance is the list of standards with which the service claims to comply. Whenever, the compliance is only partial (compliance to a specific part of a standard such as an annex or an option), the level of compliance to each standard may also be specified.
Note: Whenever a standard defines levels of compliance, this can be used in the definition to further specify the compliance degree. Compliance is self-declared. There are no certification mechanisms or authorities to verify or guarantee standards compliance. Certification is the topic of a complementary NFP. Standards compliance can be expressed as a list of tuples with three components, one to specify the name of the standard, a second to specify the version, and third to specify the level of compliance.

Relevance: "The great thing about standards is that there are so many to choose from". Many SOA-enabling standards (e.g., standards for messaging; service description and discovery; and implementation) are available for developers to build interoperable services and composite applications. Standards not only reduce efforts to use new services but also provide certain aspects of user requirements. Therefore, there is a need for service providers to specify the standards that they have chosen to implement, the version, and exactly to which part they comply. The service consumers should not go through the burden of testing the standards compliance of the services.

Measurable: Can be done through certification or observation.

10. Failure Modes are the possible ways in which service failures may occur and should be handled.

Note: Services have to enumerate the failures that can happen and the required consumer behavior for each. Examples: The database is locked by another process. Try again after a random back off period (or re-invoke the service, or roll back, or contact the customer service, etc.).

The following properties need to be captured: 1 - the failure (explanation of the exception that caused a failure such as "Bad Input Format", "Required Resource is Unavailable", "Right Access Denied", etc.) and 2- the recovery type. Such types include a) whether the service is idempotent (e.g., a service when invoked multiple times, has no further effect on its subject after the first time it is performed), and b) the capability to rollback (i.e., to maintain data integrity, it is important to detect faults before they become failures and to roll back to a sane state). This can help guarantee that the service returns to a sane state but may require allocating the responsibility for resubmission of inputs to the service consumer).

Relevance: Service consumers must anticipate that their provider will, from time to time, crash (preferably cleanly) without the opportunity for sophisticated failure handling (i.e., perhaps because of a loss of power to the computer running the provider). Consumers must therefore already have the capability of handling such a crash. Moreover, knowing the failure mode enables the service consumer to determine which uses of the service are suitable and which ones are not.

Measurable: This could be tested.

11. Transactional Service indicates whether the service can participate in transactional commits to maintain the integrity of data/solution. If a transaction is not commit- 
ted, all intermediate effects of the transaction shall be rolled-back.

Note: Transactional services require extra memory since they need to preserve the original states until they are notified to rollback or commit their changes.

Relevance: We may need to have an NFP that specifies whether a service can participate in transactional twophase commits. This involves a sequence of independent updates that must be committed as a logical unit.

Measurable: Observable.

12. Security is the protective measures that ensure a state of inviolability from hostile acts or influences.

Note: In service descriptions, service providers should state their guarantees (i.e., the famous Domino's Guarantee - Your Pizza in 30 Minutes Or It's Free) and their conditions (e.g., the responsibilities of the service consumers such as for banking services: signing out and closing the Internet browser at the end of each service invocation). The service description should indicate whenever it does not increase threats to the service security whether it supports the following functionalities:

(1) Data Privacy and Confidentiality: whether it is stored or in-transit, data should be kept private and cannot be viewed by unauthorized individuals. The service provider should state the mechanisms that ensure data privacy and confidentiality (i.e., the use of specific encryption technology).

(2) Authentication of External Service Providers: for services offered by third parties, the service should support the authentication of the service providers.

(3) Auditing: the availability of the auditing capability of service invocations that can be traced to specific users for logging and repudiation.

(4) Global Signoff: there should not be any token left in the cache memory (if any) after users exit the system.

(5) Password Policies: access management policies (i.e., password aging, password history, reminder hint or reset, password complexity, password lockout, and authentication to external authorities).

(6) Non-Repudiation: mechanisms to be able to check who did what (i.e., ability to prove that a transaction originated from a particular party, so that this party cannot deny he/she performed a certain transaction).

(7) The ability to delegate security to specialized components/services.

(8) Perimeter security requirements: a set of physical security and programmatic security policies that provide levels of protection against remote malicious activity.

(9) Protection of resources involved in service delivery.

Relevance: Service consumers need to know the security mechanisms of each service to reason about their holistic security approach for their composite services (e.g., application).

Measurable: Could be based on certifications or reputation.

13. Jurisdiction is a list of countries/territories for which a service complies with national/territorial regulations.
Note: With the ever increasing need for systems to comply with regulatory legislation, services should expose their countries/territories of jurisdiction to reflect the presumably respected legal obligations (e.g., privacy laws). This NFP is complementary to the standards compliance.

Relevance: For ethics, law, and tax purposes.

Measurable: Could be based on certification or reputation.

14. Service Versioning is the process of documenting the nature of changes for a service (i.e., interface update, backward compatibility, added or removed functionalities, or fixed bugs).

Note: Service versioning allows service consumers to compare two consecutive versions of a service and decide which version they should be using. The nature of change should be specified (1) whether we change or preserve the same interface when the service is updated; (2) the possibility of backward compatibility; (3) list of the removed functionalities; (4) list of the extensions or modification in service functionalities; and/or (5) bug fixes.

Relevance: To know how to handle future updates, i.e., simply invoke the service or look for new updates/versions.

Measurable: This is not measurable; we can simply invoke the service and observe its behavior.

15. Resource Requirements are the minimum and/or the recommended sets of requirements needed at the consumers' end to support successful execution of a service.

Note: Resources requirements may include but are not restricted to hardware (including PCs), systems and applications software, telecommunications (voice and data) and critical files.

Relevance: Service consumers should be in a position to ensure that they have the required resources before invoking any given service.

Measurable: By monitoring resources usage with a set of counters of each required resource.

16. Scalability is the property of handling increasing service invocations attempts and could be measured as the maximum number of service requests that can be executed at the same time.

Note: Scalability of a given service could be significantly impacted by utilization of other services on the same set of resources. Scalability is tightly coupled with response time. Scalability could be expressed as a natural number.

Relevance: Service consumers may need to know whether a given service can scale to increasing demand.

Measurable: By monitoring how well increasing demand is handled.

17. Server Location is a particular place in the physical space.

Note. For distributed and mirrored servers, a list of countries with territories will be provided. However, there is no guarantee the data does not transit through 
different countries. The network topology and routing are out of the control of the service provider.

Relevance: This is might be useful for consumers who do not want their data to enter a given country.

Measurable: not measurable.

\section{ADDITIONAL OBSERVATIONS}

\section{A. Groups of NFPs}

Several participants highlighted the importance of grouping these non-functional properties in some categories or subgroups, in order to make them more easily digestible by readers. We propose to divide our NFPs into four categories:

(a) Internal service appreciation: Price, resources requirements, server location, accuracy, scalability, jurisdiction, and standards compliance.

(b) External service appreciation: Reputation, certification, and usability.

(c) Service composition and invocation: Service versioning, transactional service, and failure mode.

(d) Critical service requirements: Response time, availability, reliability, and security.

Note that jurisdiction and standards compliance requirements could also be considered as external service appreciation properties that satisfy either legal or economic constraints.

\section{B. NFPs Relevant to Service Providers}

We should keep in mind that other NFPs are indeed needed and even really important from the service provider's side but less important for describing services from the consumer's perspective. For example:

Maintainability (analyzability, changeability, stability and testability): service consumers invoke the services as they are, without having access to their implementation details (i.e., in SOA services are opaque). Hence they are not supposed to do any maintenance or to be even informed about this matter.

Interoperability: SOA and its enabling technologies bring with them the promise of seamless and agile integration and interoperability, as well as cost saving due to the reuse capabilities. Whether interoperability means integrating legacy systems or enabling new business models with partners and customers, using standards is a must for SOA-based applications and it is the key for interoperability. This NFP is covered to some extent by the standards compliance NFP and hence does not really need to be exposed on its own.

Portability (adaptability, instability, and coexistence): In SOA, portability has become less of an issue than for conventional software applications. If proper architectural designs are in place, platform-specific applications can be transformed into less technology-dependent and more accommodating to interoperable business needs. This NFP is also covered by the standards compliance NFP, to some extent.

Suitability: the service consumer may end-up using a service in a very creative manner, such that even the service provider never thought that its service would be used in that given workflow and context. Hence, this criterion is not really relevant for exposition in the service description.

Controllability: the service consumer is not supposed to be controlling the service that he is invoking. The only case where a minimum controllability is needed is when something goes wrong with the service. In this case, the failure mode NFP will optimistically dictate what the service consumer should do.

A complete list is however outside the scope of our work.

\section{CONCLUSIONS AND FUTURE WORK}

The aim of this paper is to assess the relevance of NFPs for SOA and to investigate, in particular, the properties that atomic services should expose as part of their service description to empower service consumers (as opposed to providers). To meet this objective, we have performed a literature review that helped define an initial catalogue of 19 NFPs, which was hence distributed as part of an online survey. The answers and comments received from 29 knowledgeable participants from many domains helped refine this list by removing 5 of the initial NFPs, adding 3 more, and revisiting the scope, terminology, and definitions of the remaining ones. The new catalogue was further sent for inspection and comments to a subset of eight of the initial survey participants.

For each of the resulting 17 NFPs, we provided a concise definition, provided additional notes, justified its relevance, and described how to measure it. This new catalogue is important because, unlike what can be found in the literature, it focuses on the customer's perspective, it was validated with real users and experts, and it was defined in a domain-independent and technologyindependent way so that it can now be formalized and used by multiple developers and standardization bodies.

We are aware that this NFP catalogue could be debated indefinitely. We tried to motivate participation in the survey as much as possible. We need to freeze this catalogue at this point to be able to progress and enable advanced applications such as service monitoring, comparison and substitution. However, such a catalogue must also be implemented in an extensible way, so that other NFPs, perhaps more domain-dependent, can eventually be incorporated. Future work that needs to be done includes the definition of an ontology, metamodel, or data structure that will formalize precisely how each NFP can be described and measured, and how the NFPs actually relate to each other. How NFPs of composite services can be computed from the NFPs of atomic services in a context of service selection/composition and dynamic adaption is also of high interest [31]. Integration of service descriptions to existing technologies (e.g., as a complement to web service description languages) is also currently under study.

\section{ACKNOWLEDGMENT}

The authors wish to thank the anonymous survey participants, as well as Chris Hobbs and the students of the ASERT laboratory for useful discussions. This work was supported in part by a grant from NSERC. 


\section{REFERENCES}

[1] OASIS, Reference Model for Service Oriented Architecture 1.0. October 2006. http://www.oasis-open.org/ specs/index.php\#soa-rmv1.0 (last accessed: July 23, 2011)

[2] ISO, Software Engineering - Product Quality - Part 1: Quality Model. ISO/IEC 9126-1, June 2001.

[3] Organization for the Advancement of Structured Information Standards (OASIS). http://www.oasis-open. org/

[4] World Wide Web Consortium (W3C). http://www.w3.org/

[5] International Telecommunication Union (ITU). http://www.itu.int

[6] Object Management Group (OMG). http://www.omg.org/

[7] OMG, Handling of Non-Functional Properties in SOA. Draft Request for Information, Telecommunication Platform Special Interest Group, October 2008. http://telecom.omg.org/

[8] E. Kim and Y. Lee, Quality Model for Web Services v2.0, Committee Draft, OASIS, September 2005. http://www. oasis-open.org/committees/tc_home.php?wg_abbrev= wsqm (last accessed: July 23, 2011)

[9] E. Kim et al., Web Services Quality Factors Version 1.0. Committee Draft 02, OASIS, July 2010. http://docs.oasisopen.org/wsqm/wsqf/v1.0/WS-Quality-Factors-v1.0cd02.html (last accessed: July 23, 2011)

[10] S.W. Choi, J.S. Her, and S.D. Kim, "QoS Metrics for Evaluating Services from the Perspective of Service Providers", in IEEE International Conference on e-Business Engineering (ICEBE 2007). IEEE CS, pp. 622-625, 2007. doi:10.1109/ICEBE.2007.107

[11] M. Galster and E. Bucherer, "A Taxonomy for Identifying and Specifying Non-functional Requirements in Serviceoriented Development", in Proceeding 2008 IEEE Congress on Services (SERVICES 2008: Part 1), IEEE CS, pp. 345-352, 2008. doi:10.1109/SERVICES-1.2008.51

[12] Z. Balfagih, M.F. Hassan, and M.F. "Quality Model for the web services from multi-stakeholders' perspective", in Information Management and Engineering (ICIME'09), pp. 287-291, Kuala Lumpur, Malaysia, 2009. doi:10.1109/ICIME.2009.11.

[13] V. Tosic, B. Esfandiari, B. Pagurek, and K. Patel, "On requirements for ontologies in management of web services", in Int. Workshop on Web Services, e-Business, and the Semantic Web (WES'02), LNCS 2512, Springer, pp. 237-247, 2002. doi: 10.1007/3-540-36189-8_19

[14] OWL-S: Semantic Markup for Web Services. www.daml.org/services/owl-s/1.0/owl-s.html (last accessed: July 23, 2011)

[15] A.S. Vedamuthu et al., Web services policy framework (WS-Policy), version 1.5, W3C, September 2007. http://www.w3.org/TR/ws-policy/ (last accessed: July 23, 2011)

[16] Z. Chen, C. Lianf-Tien, B. Silverajan, and L. Bu-Sung, "UX - an architecture providing QoS-aware and federated support for UDDI", in Proc. 2003 Int. Conf. on Web Services (ICWS'03), Las Vegas, USA, June 2003. CSREA Press, pp. 171-176.

[17] D. Roman, H. Lausen and U. Keller, Web Service Modeling Ontology (WSMO), WSMO Working Draft, February 2007. http://www.wsmo.org/TR/d2/v1.4/ (last accessed: July 23, 2011)

[18] L. Zeng, B. Benatallah, A.H.H. Ngu, M. Dumas, J. Kalagnanam, and H. Chang, "QoS-aware middleware for Web services composition". IEEE Transactions on Software Engineering, Vol. 30(5), pp. 311-327, 2004. doi:10.1109/TSE.2004.11
[19] G. Dobson, S. Hall, and G. Kotonya, "A DomainIndependent Ontology for Non-Functional Requirements", in IEEE Int. Conf. on e-Business Engineering (ICEBE) Hong Kong. IEEE CS, 2007, pp. 563-566. doi: 10.1109/ICEBE.2007.76

[20] V. Tosic, B. Pagurek, K. Patel, B. Esfandiari, and W. Ma, "Management applications of the web service offerings language (WSOL)". Inf. Syst. 30, 7, November 2005, pp. 564-586. doi:10.1016/j.is.2004.11.005

[21] Defense Advanced Research Projects Agency (DARPA), DAML Ontology Library. http://www.daml.org/ontologies/ (last accessed: July 23, 2011)

[22] Cycorp Inc., OpenCyc Selected Vocabulary and Upper Ontology: Quantity. December 2002. http://www.cyc.com/ cycdoc/vocab/quantity-vocab.html (last accessed: July 23, 2011)

[23] J. Heflin, SHOE (Simple HTML Ontology Extensions): Measurement Ontology, version 1.0 (draft), April 2000. http://www.cs.umd.edu/projects/plus/SHOE/onts/measure1 .0.html (last accessed: July 23, 2011)

[24] D. Edmond, J. O'Sullivan, and A.H.t. Hofstede, "Two Main Challenges in Service Description: Web Service Tunnel Vision and Semantic Myopia", in W3C Workshop on Frameworks for Semantics in Web Services, Innsbruck, Austria, June 2005. http://www.w3.org/2005/04/FSWS/ Submissions/8/OSullivan_-_FSWS_-_Position_Paper.html (last accessed: July 23, 2011)

[25] J. O'Sullivan, D. Edmond, D., and A.H.t. Hofstede, "Formal description of non-functional service properties". Technical FIT-TR-2005-01, Queensland University of Technology, Brisbane (2005). http://www.wsmo.org/ papers/OSullivanTR2005.pdf (last accessed: July 23, 2011)

[26] H. Ludwig, A. Keller, A. Dan, R.P. King, and R. Franck, "Web Service Level agreement (WSLA) language specification", version 1.0, January 2003. http://www.research. ibm.com/wsla/WSLASpecV1-20030128.pdf (last accessed: July 23, 2011)

[27] A. Sahai, A. Durante, and V. Machiraju, "Towards automated SLA management for web services", Research Report HPL-2001-310 (R.1), HP Laboratories, Palo Alto, July 2002. http://www.hpl.hp.com/techreports/2001/HPL-2001310R1.pdf (last accessed: July 23, 2011)

[28] D.D. Lamanna, J. Skene, and W. Emmerich, "SLAng: a language for defining service level agreements", in Ninth IEEE Workshop on Future Trends in Distributed Computing Systems (FTDCS), Puerto Rico, May 2003. IEEE-CS Press, pp. 100-106. doi:10.1109/FTDCS.2003.1204317

[29] K. Czajkowski, A. Dan, J. Rofrano, S. Tuecke, and M. Xu, "Agreement-based grid service management (OGSIAgreement)", Version 0, Global Grid Forum, June 2003. http://www.globus.org/alliance/publications/papers/OGSI Agreement_2003_06_12.pdf (last accessed: July 23, 2011)

[30] H. Becha and D. Amyot, Non-Functional Properties of Services in SOA - Consumer's Perspective. Survey questionnaire, 2010. http://www.eecs.uottawa.ca/ damyot/pub/NFP4SOA/

[31] H. Becha, G. Mussbacher, and D. Amyot, "Modeling and Analyzing Non-Functional Requirements in Service Oriented Architecture with the User Requirements Notation", in Non-functional Properties in Service Oriented Architecture: Requirements, Models and Methods, IGI Global, USA, pp. 48-72, 2011. doi:10.4018/978-1-60566-7942.ch003 


\section{APPENDIX A: ABBREVIATIONS AND ACRONYMS}

B2B Business to Business

DAML-S DARPA Agent Markup Language

DARPA Defense Advanced Research Projects Agency

GPS

IEC

ISO

ITU

NFP

OASIS

OMG

OWL-S

QoS

RFI

SDO

SIG

SLA

SOA

UDDI

UX

W3C

WS

WSDL

WSMO

WSOL

WS-Policy

XML
Global Positioning System

International Electrotechnical Commission

International Organization for Standardization

International Telecommunication Union

Non-Functional Property

Organization for the Advancement of Structured

Information Standards

Object Management Group

Semantic Markup for Web Services

Quality of Service

Request For Information

Standards Developing Organizations

Special Interest Group

Service Level Agreement

Service Oriented Architecture

Universal Description, Discovery and Integration

UDDI eXtension

World Wide Web Consortium

Web Service

Web Services Description Language

Web Service Modeling Ontology

Web Service Offerings Language

Web Services Policy

Extensible Markup Language

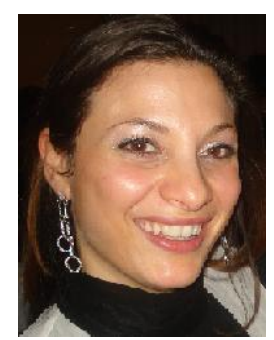

Hanane Becha received a M.Sc. degree in the area of distributed computing from the University of Ottawa in 2004. While working as a key member of the Nortel Strategic Standards development team, leading the creation of Nortel-wide standstandards strategy for SOA and Web Services and contributing to the development of SOA-related international standards, she focused on identifying and filling gaps in standards for using SOA principles in telecommunications and mission critical applications. Hanane led the Object Management Group (OMG) Telecom Special Interest Group initiative and she is the editor of multiple documents at the International Telecommunications Union, Standardization Sector (ITU-T). For her PhD studies at the University of Ottawa, Hanane is developing a framework to better handle nonfunctional properties (NFP) of services from the perspective of service consumers, to enable advanced applications such as NFP-aware service selection. Hanane also taught several courses at the University of Ottawa.

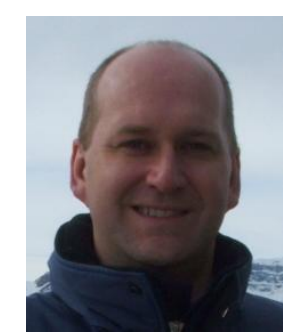

Daniel Amyot received both his Ph.D. (2001) and M.Sc. (1994) degrees in computer science from the University of Ottawa. After working for Mitel Networks as a senior researcher, he joined the School of Electrical Engineering and Computer Science of the University of Ottawa, where he is now Associate Professor in software engineering. His research interests include goal-oriented and scenario-based software engineering, requirements engineering, business process modeling, aspect-oriented modeling, and healthcare informatics. He has published over 100 papers in various conferences and in journals such as Requirements Engineering, Computer Networks, and the International Journal of Electronic Business.

Dr. Amyot is a member of ACM and IEEE Computer Society, and he is a professional engineer in the province of Québec (Canada). He is also Associate Rapporteur for requirements languages at the International Telecommunication Union, where he leads the evolution of the User Requirements Notation. 